\title{
ON THE NUMBER OF SOLUTIONS OF DIOPHANTINE EQUATIONS
}

\author{
MARTIN DAVIS ${ }^{1}$
}

ABSTRaCr. For any nontrivial set of cardinal numbers $\leqq \aleph_{0}$, it is shown that there is no algorithm for testing whether or not the number of positive integer solutions of a given polynomial Diophantine equation belongs to the set.

We consider decision problems concerning the number of distinct solutions in positive integers of polynomial Diophantine equations.

For any polynomial $P\left(x_{1}, \cdots, x_{m}\right)$ with integer coefficients (not identically zero) we let $\#(P)$ be the number of distinct positive integer solutions of

$$
P\left(x_{1}, \cdots, x_{m}\right)=0 .
$$

Thus, $0 \leqq \#(P) \leqq \aleph_{0}$. Let $C=\left\{0,1,2,3, \cdots, \aleph_{0}\right\}$. Then for given $A \subseteq C$, we may seek algorithms for testing whether or not $\#(P) \in A$. We shall prove:

TheOREM. For no subset $A \subseteq C$ (except for the trivial cases $A=\varnothing$, $A=C)$ is there an algorithm for testing whether or not $\#(P) \in A$.

In proving the theorem we shall find the following transformations useful:

(i) $T^{\infty} P=u P$ where $u$ is a variable not occurring in $P$.

(ii) $T^{+} P=P \cdot\left[\left(x_{1}-a_{1}\right)^{2}+\cdots+\left(x_{n}-a_{n}\right)^{2}\right]$ where $P=P\left(x_{1}, \cdots, x_{n}\right)$ and $\left(a_{1}, \cdots, a_{n}\right)$ is the first $n$-tuple of positive integers in some fixed (recursive) ordering which is not a solution of $P=0$. Thus,

$$
\begin{aligned}
& T^{+} P=0 \Leftrightarrow P=0 \vee\left(x_{1}, \cdots, x_{n}\right)=\left(a_{1}, \cdots, a_{n}\right) . \\
& T^{-} P=P^{2}+\prod_{i=1}^{n}\left(\sum_{j=1}^{i-1}\left(x_{j}-a_{j}\right)^{2}+\left(\left(x_{i}-a_{i}\right)^{2}-u\right)^{2}\right)^{2},
\end{aligned}
$$

where $P=P\left(x_{1}, \cdots, x_{n}\right)$ and $\left(a_{1}, \cdots, a_{n}\right)$ is the first $n$-tuple of positive

Received by the editors December 1, 1971.

AMS 1970 subject classifications. Primary 02F50, 10B99, 10N05, 02E15.

Key words and phrases. Diophantine equations, unsolvable problems, Hilbert's tenth problem, recursive functions.

1 This work was supported by the National Science Foundation. 
integers (using the same ordering as in (ii)) which is a solution of $P=0$; if $P=0$ has no solutions, $T^{-} P$ is not defined. ${ }^{2}$ Thus,

$$
T^{-} P=0 \Leftrightarrow P=0 \&\left(x_{1}, \cdots, x_{n}\right) \neq\left(a_{1}, \cdots, a_{n}\right) .
$$

We have at once:

$$
\#\left(T^{\infty} P\right)=\aleph_{0} \Leftrightarrow \#(P)>0 .
$$

(For, any solution of $P=0$ gives rise to infinitely many solutions as $u=1,2,3, \cdots$.

$$
\begin{aligned}
& \#\left(T^{+} P\right)=\#(P)+1 \\
& \#\left(T^{-} P\right)=\#(P)-1 \quad \text { if } \#(P)>0 .
\end{aligned}
$$

Note that $T^{\infty}, T^{+}$are recursive operations and that $T^{-}$is partial recursive. We set $T^{0} P=P$ and, for $m>0, T^{m+1} P=T^{+}\left(T^{m} P\right), T^{-m-1}=T^{-(}\left(T^{-m} P\right)$. Thus, for $m>0$,

$$
\begin{aligned}
& \#\left(T^{m} P\right)=\#(P)+m, \\
& \#\left(T^{-m} P\right)=\#(P)-m \text { if } \#(P) \geqq m .
\end{aligned}
$$

We now proceed with the proof of the theorem:

Case I. $A=\{0\}$. This is just Hilbert's tenth problem, and so it is known that there is no algorithm. ${ }^{3}$

Case II. $A=\{m\}, 0<m<\aleph_{0}$. We have, for any polynomial $P$ (using (4)),

$$
\#(P)=0 \Leftrightarrow \#\left(T^{m} P\right)=m .
$$

Hence an algorithm for this case would yield one for Case I.

Case III. $A=\left\{\boldsymbol{\aleph}_{0}\right\}$. For any polynomial $P$,

$$
\#(P)=0 \Leftrightarrow \#\left(T^{\infty} P\right) \neq \aleph_{0} .
$$

So again an algorithm for this case would yield one for Case I.

Case IV. $\boldsymbol{\aleph}_{0} \in A, 0 \notin A, A \neq\left\{\boldsymbol{\aleph}_{0}\right\}$. Let $m$ be the least element of $A$. Then for any $P$,

$$
\#(P)=m \Leftrightarrow \#(P) \in A \& \#\left(T^{\infty} T^{\cdots m} P\right) \notin A .
$$

So an algorithm for this case would yield one for Case II.

\footnotetext{
${ }^{2}$ My original definition of $T^{-}$was faulty. I am grateful to Yuri Matijasevič for this correction. I am also grateful to N. K. Kosovskii and to the referee for pointing out oversights.

${ }^{3}$ This was shown in Matijasevič [2]. Cf. also [1]. For historical remarks and further references, cf. [3].
} 
Case V. $\aleph_{0} \notin A, 0 \in A, A \neq\{0\}$. Then $C-A$ is in Case III or IV.

Case VI. $0 \notin A, \aleph_{0} \notin A, A$ contains at least 2 elements. Let $m$ be the least element of $A$ and let $B=\{x-m \mid x \in A\}$. Then $B$ is in Case $\mathrm{V}$, and

$$
\#(P) \in B \Leftrightarrow \#\left(T^{m} P\right) \in A
$$

so that an algorithm for $A$ would yield one for $B$.

Case VII. $0 \in A, \aleph_{0} \in A, A \neq C$. Then $C-A$ is in Case II or VI.

Since Cases I through VII exhaust all possibilities, this proves the theorem.

\section{REFERENCES}

1. Martin Davis, An explicit Diophantine definition of the exponential function, Comm. Pure Appl. Math. 24 (1971), 137-145. MR 42 \#7632.

2. Ju. V. Matijasevič, Enumerable sets are Diophantine, Dokl. Akad. Nauk SSSR 191 (1970), 279-282=Soviet Math. Dokl. 11 (1970), 354-358.

3. Julia Robinson, Hilbert's tenth problem, Proc. Sympos. Pure Math., vol. 20, Amer. Math. Soc., Providence, R.I., 1971, pp. 191-194.

Courant Institute of Mathematical Sciences, New York University, New YORK, NEW YORK 10012 